\title{
Corrigendum: Protein-structure-guided discovery of functional mutations across 19 cancer types
}

Beifang Niu, Adam D Scott, Sohini Sengupta, Matthew H Bailey, Prag Batra, Jie Ning, Matthew A Wyczalkowski, Wen-Wei Liang, Qunyuan Zhang, Michael D McLellan, Sam Q Sun, Piyush Tripathi, Carolyn Lou, Kai Ye, R Jay Mashl, John Wallis, Michael C Wendl, Feng Chen \& Li Ding

Nat. Genet. 48, 827-837 (2016); published online 13 June 2016; corrected after print 20 March 2017

In the version of this article initially published, residue R384 was incorrectly highlighted in the protein model depicted in Figure 7e. The correct residue is R394. The error has been corrected in the HTML and PDF versions of the article.

\section{Corrigendum: Whole-genome sequencing identifies rare genotypes in COMP and CHADL associated with high risk of hip osteoarthritis}

Unnur Styrkarsdottir, Hannes Helgason, Asgeir Sigurdsson, Gudmundur L Norddahl, Arna B Agustsdottir, Louise N Reynard, Amanda Villalvilla, Gisli H Halldorsson, Aslaug Jonasdottir, Audur Magnusdottir, Asmundur Oddson, Gerald Sulem, Florian Zink, Gardar Sveinbjornsson, Agnar Helgason, Hrefna S Johannsdottir, Anna Helgadottir, Hreinn Stefansson, Solveig Gretarsdottir, Thorunn Rafnar, Ina S Almdahl, Anne Brækhus, Tormod Fladby, Geir Selbæk, Farhad Hosseinpanah, Fereidoun Azizi, Jung Min Koh, Nelson L S Tang, Maryams Danesphour, Jose I Mayordomo, Corrine Welt, Peter S Braund, Nilesh J Samani, Lambertus A Kiemeney, L Stefan Lohmander, Claus Christiansen, Ole A Andreassen, arcOGEN consortium, Olafur Magnusson, Gisli Masson, Augustine Kong, Ingileif Jonsdottir, Daniel Gudbjartsson, Patrick Sulem, Helgi Jonsson, John Loughlin, Thorvaldur Ingvarsson, Unnur Thorsteinsdottir \& Kari Stefansson

Nat. Genet.; doi:10.1038/ng.3816; corrected online 17 April 2017

In the version of this article initially published online, the name of author Maryam S. Daneshpour was spelled incorrectly. The error has been corrected in the print, PDF and HTML versions of this article.

\section{Erratum: Large-scale analyses of common and rare variants identify 12 new loci associated with atrial fibrillation}

Ingrid E Christophersen, Michiel Rienstra, Carolina Roselli, Xiaoyan Yin, Bastiaan Geelhoed, John Barnard, Honghuang Lin, Dan E Arking, Albert V Smith, Christine M Albert, Mark Chaffin, Nathan R Tucker, Molong Li, Derek Klarin, Nathan A Bihlmeyer, Siew-Kee Low, Peter E Weeke, Martina Müller-Nurasyid, J Gustav Smith, Jennifer A Brody, Maartje N Niemeijer, Marcus Dörr, Stella Trompet, Jennifer Huffman, Stefan Gustafsson, Claudia Schurmann, Marcus E Kleber, Leo-Pekka Lyytikäinen, Ilkka Seppälä, Rainer Malik, Andrea R V R Horimoto, Marco Perez, Juha Sinisalo, Stefanie Aeschbacher, Sébastien Thériault, Jie Yao, Farid Radmanesh, Stefan Weiss, Alexander Teumer, Seung Hoan Choi, Lu-Chen Weng, Sebastian Clauss, Rajat Deo, Daniel J Rader, Svati H Shah, Albert Sun, Jemma C Hopewell, Stephanie Debette, Ganesh Chauhan, Qiong Yang, Bradford B Worrall, Guillaume Paré, Yoichiro Kamatani, Yanick P Hagemeijer, Niek Verweij, Joylene E Siland, Michiaki Kubo, Jonathan D Smith, David R Van Wagoner, Joshua C Bis, Siegfried Perz, Bruce M Psaty, Paul M Ridker, Jared W Magnani, Tamara B Harris, Lenore J Launer, M Benjamin Shoemaker, Sandosh Padmanabhan, Jeffrey Haessler, Traci M Bartz, Melanie Waldenberger, Peter Lichtner, Marina Arendt, Jose E Krieger, Mika Kähönen, Lorenz Risch, Alfredo J Mansur, Annette Peters, Blair H Smith, Lars Lind, Stuart A Scott, Yingchang Lu, Erwin B Bottinger, Jussi Hernesniemi, Cecilia M Lindgren, Jorge A Wong, Jie Huang, Markku Eskola, Andrew P Morris, Ian Ford, Alex P Reiner, Graciela Delgado, Lin Y Chen, Yii-Der Ida Chen, Roopinder K Sandhu, Man Li, Eric Boerwinkle, Lewin Eisele, Lars Lannfelt, Natalia Rost, Christopher D Anderson, Kent D Taylor, Archie Campbell, Patrik K Magnusson, David Porteous, Lynne J Hocking, Efthymia Vlachopoulou, Nancy L Pedersen, Kjell Nikus, Marju Orho-Melander, Anders Hamsten, Jan Heeringa, Joshua C Denny, Jennifer Kriebel, Dawood Darbar, Christopher Newton-Cheh, Christian Shaffer, Peter W Macfarlane, Stefanie Heilmann-Heimbach, Peter Almgren, Paul L Huang, Nona Sotoodehnia, Elsayed Z Soliman, Andre G Uitterlinden, Albert Hofman, Oscar H Franco, Uwe Völker, Karl-Heinz Jöckel, Moritz F Sinner, Henry J Lin, Xiuqing Guo, METASTROKE Consortium of the ISGC, Neurology Working Group of the CHARGE Consortium, Martin Dichgans, Erik Ingelsson, Charles Kooperberg, Olle Melander, Ruth J F Loos, Jari Laurikka, David Conen, Jonathan Rosand, Pim van der Harst, Marja-Liisa Lokki, Sekar Kathiresan, Alexandre Pereira, J Wouter Jukema, Caroline Hayward, Jerome I Rotter, Winfried März, Terho Lehtimäki, Bruno H Stricker, Mina K Chung, Stephan B Felix, Vilmundur Gudnason, Alvaro Alonso, Dan M Roden, Stefan Kääb, Daniel I Chasman, Susan R Heckbert, Emelia J Benjamin, Toshihiro Tanaka, Kathryn L Lunetta, Steven A Lubitz \& Patrick T Ellinor for the AFGen Consortium Nat. Genet.; 10.1038/ng.3843; corrected online 11 May 2017

In the version of this article initially published online, the authors were incorrectly defined as members of the AFGen consortium in the author list. The members of the consortium are listed in the Supplementary Note. The error has been corrected in the print, PDF and HTML versions of this article. 